\title{
Development of ceftazidime resistance in an acute Burkholderia pseudomallei infection
}

This article was published in the following Dove Press journal:

Infection and Drug Resistance

2I August 2012

Number of times this article has been viewed

\section{Derek S Sarovich ${ }^{1,2, *}$ \\ Erin P Price ${ }^{1,2, *}$ \\ Direk Limmathurotsakul ${ }^{3}$ \\ James M Cook' \\ Alex T Von Schulze' \\ Spenser R Wolken' \\ Paul Keim' \\ Sharon J Peacock ${ }^{3,4}$ \\ Talima Pearson' \\ 'Center for Microbial Genetics and Genomics, Northern Arizona University, Flagstaff, AZ, USA; \\ ${ }^{2}$ Tropical and Emerging Infectious Diseases Division, Menzies School of Health Research, Darwin, Australia; ${ }^{3}$ Department of Microbiology and Immunology, Faculty of Tropical Medicine, Mahidol University, Bangkok, Thailand; ${ }^{4}$ Department of Medicine, University of Cambridge, Cambridge, United Kingdom \\ *These authors contributed equally to this work}

Correspondence: Talima Pearson Center for Microbial Genetics and Genomics, ARD BIdg 56, 3rd Floor, I298 S Knoles Dr, Flagstaff, AZ 86011 , USA

Tel +l 9285234290

$\mathrm{Fax}+19285234015$

Email talima.pearson@nau.edu

\begin{abstract}
Burkholderia pseudomallei, a bacterium that causes the disease melioidosis, is intrinsically resistant to many antibiotics. First-line antibiotic therapy for treating melioidosis is usually the synthetic $\beta$-lactam, ceftazidime (CAZ), as almost all B. pseudomalle $i$ strains are susceptible to this drug. However, acquired CAZ resistance can develop in vivo during treatment with CAZ, which can lead to mortality if therapy is not switched to a different drug in a timely manner. Serial B. pseudomallei isolates obtained from an acute Thai melioidosis patient infected by a CAZ susceptible strain, who ultimately succumbed to infection despite being on CAZ therapy for the duration of their infection, were analyzed. Isolates that developed CAZ resistance due to a proline to serine change at position 167 in the $\beta$-lactamase PenA were identified. Importantly, these CAZ resistant isolates remained sensitive to the alternative melioidosis treatments; namely, amoxicillin-clavulanate, imipenem, and meropenem. Lastly, real-time polymerase chain reaction-based assays capable of rapidly identifying CAZ resistance in $B$. pseudomallei isolates at the position 167 mutation site were developed. The ability to rapidly identify the emergence of CAZ resistant $B$. pseudomallei populations in melioidosis patients will allow timely alterations in treatment strategies, thereby improving patient outcomes for this serious disease.
\end{abstract}

Keywords: Burkholderia pseudomallei, ceftazidime, antibiotic resistance, melioidosis, $\beta$-lactamase, penA

\section{Introduction}

Burkholderia pseudomallei is the etiologic agent of melioidosis, a disease endemic to Southeast Asia and northern Australia that is becoming increasingly identified in other tropical regions throughout the world. ${ }^{1}$ B. pseudomallei is intrinsically resistant to several antimicrobial agents, limiting treatment options for melioidosis. ${ }^{2,3}$ In Thailand, confirmed melioidosis cases are typically treated with intravenous ceftazidime (CAZ), a third-generation cephalosporin that is routinely administered for a minimum of 10 days, or considerably longer for complex, deep-seated infections. ${ }^{4}$ CAZ is also routinely administered for suspected melioidosis cases, and for confirmed cases prior to the availability of antimicrobial susceptibility data, on the basis that primary CAZ resistance is rare. ${ }^{2,5}$ However, recent reports have demonstrated that CAZ resistance can arise in vivo following chemotherapy, ${ }^{3,5-9}$ especially where CAZ has been administered on multiple occasions, such as in relapse melioidosis cases. $^{5}$ Early detection of $B$. pseudomallei resistance towards clinically relevant antibiotics such as CAZ is thus an important factor in reducing the morbidity and mortality of melioidosis. 
Several molecular mechanisms for CAZ resistance have been reported in the B. pseudomallei literature, all of which have been shown to arise in vivo following CAZ administration. A single-nucleotide polymorphism (SNP) in the promoter region of the $\beta$-lactamase gene, penA ( -21$)$ has been shown to cause upregulation of PenA elevating the CAZ minimum inhibitory concentration (MIC) from $1.5 \mu \mathrm{g} / \mathrm{mL}$ in wild-type $B$. pseudomallei to 6-16 $\mu \mathrm{g} / \mathrm{mL}$ in PenA -21A mutants depending on the strain. ${ }^{5,10}$ Within the penA coding sequence, amino acid substitutions at positions $69^{5,7}$ and $167^{11}$ lead to markedly increased CAZ hydrolysis compared with wild-type $B$. pseudomallei isolates (MIC values of $\geq 256 \mu \mathrm{g} / \mathrm{mL}$ and $64 \mu \mathrm{g}$ / $\mathrm{mL}$ for cysteine to tyrosine at position 69 [C69Y] and proline to serine at position 167 [P167S] mutants, respectively). Lastly, a large chromosomal deletion encompassing penicillin-binding protein-3 causes the loss of a CAZ-binding cell-surface molecule, resulting in high-level CAZ resistance (MIC $\geq 256 \mu \mathrm{g}$ ) $\mathrm{mL})^{6}$

\section{Case study}

Forty B. pseudomallei isolates obtained from a single Thai patient (P45) with acute, nonresolving melioidosis were tested for CAZ resistance. P45, a 51-year-old male rice farmer, presented with a leg abscess and high fever and was treated on admission with intravenous ceftriaxone and clindamycin for 1 day, then switched to intravenous CAZ after confirmation of B. pseudomallei by an immunofluorescence assay. ${ }^{12}$ P45 initially responded well to therapy, but after 2 weeks of treatment he had a marked deterioration associated with the development of hypotension, dyspnea, recurrence of fever, and pleural effusion on chest radiography. Despite continued CAZ therapy, P45 subsequently died. It was demonstrated previously that the $B$. pseudomallei isolates obtained from P45 were clonal. ${ }^{13}$ Thus, having ruled out coinfection or reinfection, it was suspected that in vivo development of CAZ resistance may have accounted for treatment failure in P45.

\section{Materials and methods}

\section{Ethics statement}

Informed written consent for genetic studies of these isolates was provided by the patient and approved by the Human Research Ethics Committee of the Faculty of Tropical Medicine, Mahidol University (Bangkok, Thailand). ${ }^{14}$

\section{Culture conditions and antibiotic sensitivity testing}

B. pseudomallei isolates were grown as described elsewhere. ${ }^{5}$ MIC values for CAZ, amoxicillin-clavulanate, imipenem, and meropenem were determined using Etest ${ }^{\circledR}$ (bioMerieux SA, Marcy l'Etoile, France) according to manufacturer's instructions. CAZ MIC values for susceptible, intermediate resistant, and resistant cutoffs were defined as $\leq 8 \mu \mathrm{g} / \mathrm{mL}$, $16 \mu \mathrm{g} / \mathrm{mL}$, and $\geq 32 \mu \mathrm{g} / \mathrm{mL}$, respectively. ${ }^{2}$

\section{SYBR Green-based mismatch amplification mutation assay (SYBR-MAMA)}

SYBR-MAMA polymerase chain reaction setups and thermocycling conditions were carried out as described elsewhere. ${ }^{5}$ The primers were as follows: penAP167S_ForM (5'-TTTTTTTTTTTTTTTAGCGCCGTGTTCAGCTCAtA and penAP167S_RevM (5'-GCGACGTTCCGGCTCGAT) to amplify the mutant allele; penAP167S_ForWT (5'- GCCCGCCCGCCCGCCCGCGCCGTGTTCAGCTCAt $\underline{G})$ and penAP167S_RevWT (5'-CGACGTTCCGGCTCGATC) to interrogate the wild-type SNP state. Lowercase nucleotides indicate deliberately incorporated antepenultimate mismatches $;{ }^{15}$ underlined nucleotides represent the SNP. All samples were run in duplicate, and no-template controls were included in every run.

\section{Results}

The initial 30 B. pseudomallei isolates collected upon P45's admission to hospital were sensitive to CAZ $(2 \mu \mathrm{g} / \mathrm{mL})$ as determined by Etest. However, ten isolates collected 18 days later showed a predominately CAZ-resistant collection of nine strains $(64 \mu \mathrm{g} / \mathrm{mL})$, with a single susceptible strain. Given that the CAZ-resistant strains possessed MIC values similar to those from PenAP167S-mutated B. pseudomallei strains, ${ }^{11}$ it was expected that the latter P45 B. pseudomallei isolates would possess the same penA mutation. Sanger sequencing of penA from representative sensitive and resistant isolates confirmed a P167S substitution (Ambler's numbering scheme $)^{16}$ (Figure 1).

A SYBR-MAMA ${ }^{17}$ was developed to rapidly interrogate the mutant and wild-type variants at PenA 167 in the remaining P45 isolates. The PenA 167 SYBR-MAMA readily discriminated between mutant and wild-type alleles (Figure 2). In all cases, the PenA 167 genotype corresponded with the CAZ MIC; CAZ-susceptible strains $(2 \mu \mathrm{g} / \mathrm{mL})$ exhibited preferential amplification of the wild-type allele (Figure 2, left panel) and CAZ-resistant isolates preferentially amplified the mutant PenA 167 allele (Figure 2, right panel).

It has been previously shown that upregulatory pen $A$ promoter mutations that increase CAZ resistance can also confer cross-resistance to the alternative treatments for melioidosis; namely, amoxicillin-clavulanate and 


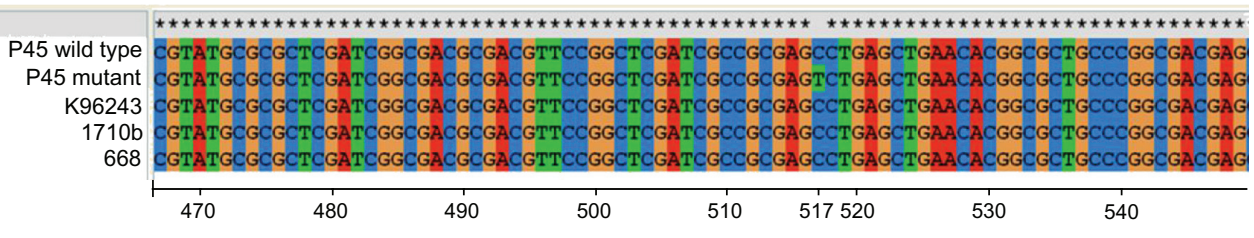

Figure I DNA alignments of penA from patient 45 wild-type and mutant alleles against three other wild-type Burkholderia pseudomallei strains - K96243, I7I0b, and 668 - showing a cytosine/thymine nucleotide transition at position 517 (according to penA annotation in B. pseudomallei K96243). ${ }^{22}$

Note: This transition results in an amino acid substitution from proline to serine at position 167 in PenA.

Abbreviation: $\mathrm{P} 45$, patient 45 .

imipenem. ${ }^{5,18}$ Therefore, the effect of the PenA P167S substitution on other $\beta$-lactam and carbapenem antibiotics commonly used to treat melioidosis was examined to determine whether this mutation also caused cross-resistance in this isolate. MIC values for amoxicillin-clavulanate, imipenem, and meropenem were determined for two CAZsusceptible and CAZ-resistant B.pseudomalle $i$ isolates from P45 using Etest. Both the wild-type and mutated isolates remained susceptible to these alternate treatment options (MIC value: meropenem $=1 \mu \mathrm{g} / \mathrm{mL}$; imipenem $=0.5 \mu \mathrm{g} / \mathrm{mL}$; amoxicillin-clavulanate $=1.5 \mu \mathrm{g} / \mathrm{mL}$ ), demonstrating that the P167S substitution had no effect on these alternate treatment strategies.

\section{Discussion}

The in vivo development of antibiotic resistance can have serious consequences on patient outcomes and is especially problematic where treatment options are already limited. For B. pseudomallei, development of resistance to the first-line antibiotic, CAZ, is becoming increasingly recognized as an important cause of treatment failure and infection relapse. Melioidosis cases reporting CAZ-resistant in vivo B. pseudomallei populations have now been described in Southeast Asia, India, Taiwan, and Australia. ${ }^{5,6,8,9}$ Given the protracted and lengthy regimen required for effective treatment of melioidosis, and the occurrence of relapse/ recrudescent cases in $\sim 6 \%$ of patients, ${ }^{19,20}$ the development of in vivo CAZ resistance is potentially more common than first thought.

In the current study, the molecular basis behind treatment failure in an acute case of melioidosis in Thailand that lead to patient mortality was identified. The P167S mutation appeared to only increase CAZ hydrolysis, with this study and others showing that ceftriaxone, imipenem, meropenem, and amoxicillin-clavulanate MIC values are unaffected by this mutation. ${ }^{11,18}$ Therefore, the development of the P167S mutation among the $\mathrm{P} 45$ in vivo $B$. pseudomallei population was most likely a result of CAZ administration. Nine out of ten colonies obtained from $\mathrm{P} 45$ following melioidosis relapse were CAZ resistant after 17 days of treatment with this drug, indicating strong positive selection of the P167S mutation under CAZ treatment.
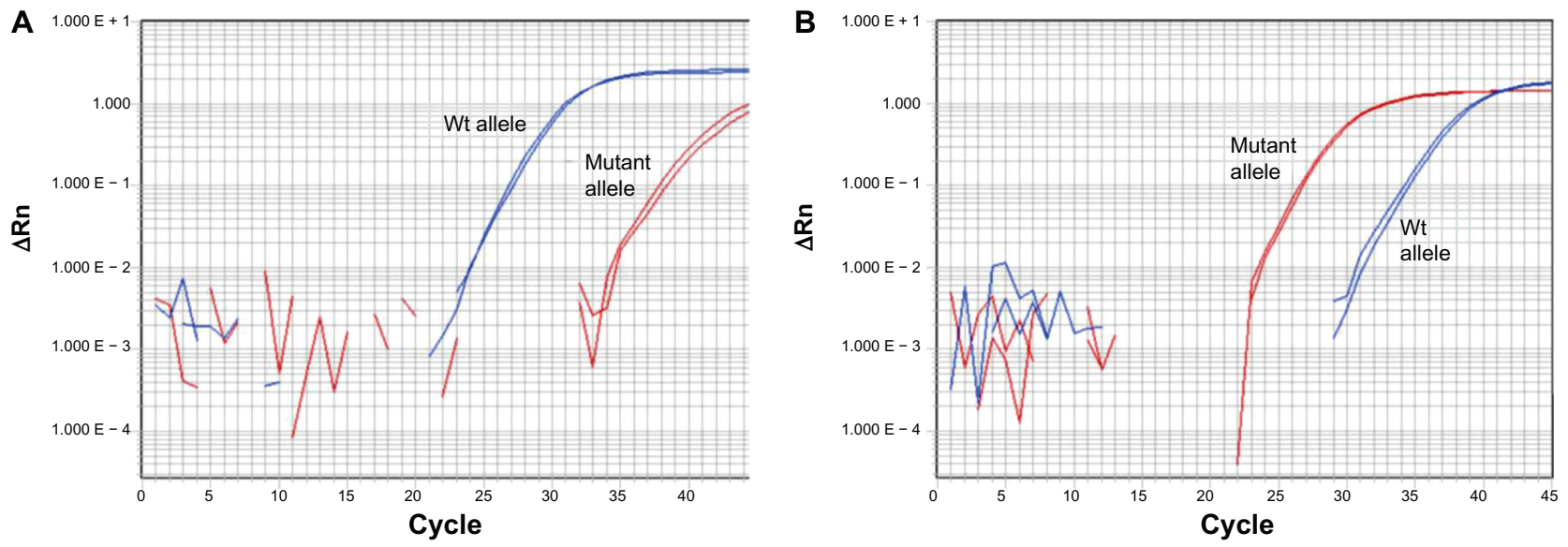

Figure 2 SYBR Green mismatch amplification mutation assay interrogation of the proline to serine at position I67 (PI67S) PenA mutation, conferred by a cytosine/ thymine nucleotide transition in penA at position 517 (according to penA annotation in Burkholderia pseudomallei K96243; ${ }^{22}$ see Figure I) in patient 45 B. pseudomallei isolates. (A) Example amplification of the wild-type PI67S penA allele. (B) Example amplification of the mutated PI67S penA allele. All mutant PI67S B. pseudomallei strains from patient 45 contained an elevated ceftazidime minimum inhibitory concentration of $64 \mu \mathrm{g} / \mathrm{mL}$ compared with the wild-type strains $(2 \mu \mathrm{g} / \mathrm{mL})$. Abbreviation: Wt, wild-type. 
Elucidation of resistance mechanisms towards clinically relevant antibiotics is crucial in determining effective treatment regimens for melioidosis. A real-time polymerase chain reaction assay that enables the rapid detection of $\mathrm{P} 167 \mathrm{~S}$ B. pseudomallei CAZ mutants in vivo is herein described. The availability of real-time assays for resistance detection allows clinicians to monitor infection dynamics and thus alter chemotherapy in real time, leading to more effective antimicrobial treatments and better patient outcomes. In addition, this study further challenges the dogma that a single isolate is representative of the in vivo bacterial population dynamics. $^{13,21}$ It was demonstrated that such practices can lead to erroneous assumptions regarding antibiotic resistance, as one out of ten isolates collected from P45 18 days after admission still possessed a CAZ-sensitive phenotype.

It is recommended that in acute nonresolving melioidosis cases, good clinical practice should entail collection and characterization of multiple clinical specimens from multiple time points, where possible, to detect for emerging antimicrobial resistance. The introduction of real-time polymerase chain reaction platforms into clinics will lead to improved therapeutic outcomes by enabling minor component resistance to be detected well before conventional culture-based techniques (eg, Etest) can identify resistance.

\section{Acknowledgment}

This work was supported by the US Department of Homeland Security via award HSHQDC-10-C-00139.

\section{Disclosure}

The authors report no conflicts of interest in this work.

\section{References}

1. Currie BJ, Dance DA, Cheng AC. The global distribution of Burkholderia pseudomallei and melioidosis: an update. Trans R Soc Trop Med Hyg. 2008;102(Suppl 1):S1-S4.

2. Wuthiekanun V, Amornchai P, Saiprom N, et al. Survey of antimicrobial resistance in clinical Burkholderia pseudomallei isolates over two decades in Northeast Thailand. Antimicrob Agents Chemother. 2011; 55(11):5388-5391.

3. Jenney AW, Lum G, Fisher DA, Currie BJ. Antibiotic susceptibility of Burkholderia pseudomallei from tropical northern Australia and implications for therapy of melioidosis. Int J Antimicrob Agents. 2001; 17(2):109-113.

Infection and Drug Resistance

\section{Publish your work in this journal}

Infection and Drug Resistance is an international, peer-reviewed openaccess journal that focuses on the optimal treatment of infection (bacterial, fungal and viral) and the development and institution of preventive strategies to minimize the development and spread of resistance. The journal is specifically concerned with the epidemiology of antibiotic

Submit your manuscript here: http://www.dovepress.com/infection-and-drug-resistance-journal
4. Cheng AC, Currie BJ. Melioidosis: epidemiology, pathophysiology, and management. Clin Microbiol Rev. 2005;18(2):383-416.

5. Sarovich DS, Price EP, Von Schulze AT, et al. Characterization of ceftazidime resistance mechanisms in clinical isolates of Burkholderia pseudomallei from Australia. PLoS One. 2012;7(2):e30789.

6. Chantratita N, Rholl DA, Sim B, et al. Antimicrobial resistance to ceftazidime involving loss of penicillin-binding protein 3 in Burkholderia pseudomallei. Proc Natl Acad Sci U S A. 2011;108(41):17165-17170.

7. Sam IC, See KH, Puthucheary SD. Variations in ceftazidime and amoxicillin-clavulanate susceptibilities within a clonal infection of Burkholderia pseudomallei. J Clin Microbiol. 2009;47(5): $1556-1558$.

8. Kung CT, Lee CH, Li CJ, Lu HI, Ko SF, Liu JW. Development of ceftazidime resistance in Burkholderia pseudomallei in a patient experiencing melioidosis with mediastinal lymphadenitis. Ann Acad Med Singapore. 2010;39(12):945-947.

9. Behera B, Prasad Babu T, Kamalesh A, Reddy G. Ceftazidime resistance in Burkholderia pseudomallei: first report from India. Asian Pac J Trop Med. 2012;5(4):329-330.

10. Hayden HS, Lim R, Brittnacher MJ, et al. Evolution of Burkholderia pseudomallei in recurrent melioidosis. PLoS One. 2012;7(5):e36507.

11. Tribuddharat C, Moore RA, Baker P, Woods DE. Burkholderia pseudomallei class A $\beta$-lactamase mutations that confer selective resistance against ceftazidime or clavulanic acid inhibition. Antimicrob Agents Chemother. 2003;47(7):2082-2087.

12. Walsh AL, Smith MD, Wuthiekanun V, et al. Immunofluorescence microscopy for the rapid diagnosis of melioidosis. J Clin Pathol. 1994; 47(4):377-379.

13. Price EP, Hornstra HM, Limmathurotsakul D, et al. Within-host evolution of Burkholderia pseudomallei in four cases of acute melioidosis. PLoS Pathog. 2010;6(1):e1000725.

14. Limmathurotsakul D, Wuthiekanun V, Chantratita N, et al. Simultaneous infection with more than one strain of Burkholderia pseudomallei is uncommon in human melioidosis. J Clin Microbiol. 2007;45(11): 3830-3832.

15. Hezard N, Cornillet P, Droulle C, Gillot L, Potron G, Nguyen P. Factor V Leiden: detection in whole blood by ASA PCR using an additional mismatch in antepenultimate position. Thromb Res. 1997;88(1):59-66.

16. Ambler RP, Coulson AF, Frere JM, et al. A standard numbering scheme for the class A $\beta$-lactamases. Biochem J. 1991;276(Pt 1):269-270.

17. Germer S, Higuchi R. Single-tube genotyping without oligonucleotide probes. Genome Res. 1999;9(1):72-78.

18. Rholl DA, Papp-Wallace KM, Tomaras AP, Vasil ML, Bonomo RA, Schweizer HP. Molecular investigations of PenA-mediated $\beta$-lactam resistance in Burkholderia pseudomallei. Front Microbiol. 2011; 2:139.

19. Currie BJ, Ward L, Cheng AC. The epidemiology and clinical spectrum of melioidosis: 540 cases from the 20 year Darwin prospective study. PLoS Negl Trop Dis. 2010;4(11):e900.

20. Limmathurotsakul D, Chaowagul W, Chantratita N, et al. A simple scoring system to differentiate between relapse and re-infection in patients with recurrent melioidosis. PLoS Negl Trop Dis. 2008;2(10):e327.

21. Pearson T, U'Ren JM, Schupp JM, et al. VNTR analysis of selected outbreaks of Burkholderia pseudomallei in Australia. Infect Genet Evol. 2007;7(4):416-423.

22. Holden MT, Titball RW, Peacock SJ, et al. Genomic plasticity of the causative agent of melioidosis, Burkholderia pseudomallei. Proc Natl Acad Sci U S A. 2004;101(39):14240-14245.

\section{Dovepress}

resistance and the mechanisms of resistance development and diffusion in both hospitals and the community. The manuscript management system is completely online and includes a very quick and fair peerreview system, which is all easy to use. Visit http://www.dovepress.com/ testimonials.php to read real quotes from published authors. 